\title{
A proof of the smoothing properties of the positive part of Boltzmann's kernel
}

François Bouchut and Laurent Desvillettes

Abstract. We give two direct proofs of Sobolev estimates for the positive part of Boltzmann's kernel. The first deals with a cross section with separated variables; no derivative is needed in this case. The second is concerned with a general cross section having one derivative in the angular variable.

Résumé. Nous donnons deux preuves directes des estimations de Sobolev pour la partie positive du noyau de Boltzmann. La première concerne les sections efficaces à variables séparées; aucune dérivée n'est nécessaire dans ce cas. La deuxième traite des sections efficaces générales ayant une dérivée dans la variable angulaire.

\section{Introduction.}

The Boltzmann quadratic kernel $Q$ models binary collisions occurring in a rarefied monatomic gas ( $c f .[3],[4],[9])$. It can be written under the form

$$
Q(f)(v)=Q^{+}(f)(v)-f(v) L f(v)
$$

where $L f$ is a linear convolution operator, and $Q^{+}$is the positive part 
of $Q$, defined by

$$
\begin{gathered}
Q^{+}(f)(v)=\iint_{\substack{v_{*} \in \mathbb{R}^{N} \\
\sigma \in S^{N-1}}} f\left(\frac{v+v_{*}}{2}-\frac{\left|v-v_{*}\right|}{2} \sigma\right) f\left(\frac{v+v_{*}}{2}+\frac{\left|v-v_{*}\right|}{2} \sigma\right) \\
\cdot B\left(\left|v-v_{*}\right|, \frac{v-v_{*}}{\left|v-v_{*}\right|} \cdot \sigma\right) d \sigma d v_{*} .
\end{gathered}
$$

The nonnegative cross section $B$ depends on the type of interaction between the particles of the gas.

In a gas in which particles interact with respect to forces proportional to $r^{-s}, s \geq 2$, the cross section $B$ writes

$$
B(x, u)=b(x) \beta(u),
$$

where

$$
b(x)=x^{(s-5) /(s-1)},
$$

and $\beta$ has a strong singularity near $u=1$.

The classical assumption of angular cutoff of Grad [6] (that is $\beta \in$ $\left.L^{1}(]-1,1[)\right)$ is used to remove this singularity. It will always be made in this paper. To get an idea of the properties of $Q$ when this assumption is not made, we refer the reader for example to [5] or [8].

The properties of $Q^{+}$(with the assumption of angular cutoff of Grad) have first been investigated by P.-L. Lions in [7]. In this work, it is proved that if $B$ is a very smooth function with support avoiding certain points, then there exists $C_{N, B}$ such that

$$
\left\|Q^{+}(f)\right\|_{\dot{H}^{(N-1) / 2}\left(\mathbb{R}_{v}^{N}\right)} \leq C_{N, B}\|f\|_{L^{1}\left(\mathbb{R}_{v}^{N}\right)}\|f\|_{L^{2}\left(\mathbb{R}_{v}^{N}\right)}
$$

for any $f \in L^{1} \cap L^{2}\left(\mathbb{R}_{v}^{N}\right)$.

The proof of this estimate used the theory of Fourier integral operators. The very restricting conditions on $B$ were not a serious inconvenience since in the application to the inhomogeneous Boltzmann equation, only the strong compactness in $L^{1}$ of $Q^{+}(f)$ was used, and not the estimate itself, so that these smoothness assumptions could be relaxed by suitable approximations of $B$. Notice that the use of the Fourier transform in the velocity variable in the context of the Boltzmann equation was already used by Bobylev in [2]. 
An extension of this work to the case of the relativistic Boltzmann kernel can be found in [1].

Then, another proof of (1.5) was given by Wennberg [10] with the help of the regularizing properties of the (generalized) Radon transform. The hypothesis on $B$ were considerably diminished, so that for example forces in $r^{-s}$ with angular cutoff and $s \geq 9$ were included. Considerations on related kernels (for example the relativistic kernel) can also be found in [11].

This work is intended to give yet another proof of (1.5)-like estimates, using only elementary properties of the Fourier transform. Moreover, we prove that the estimate holds for a large class of cross sections $B$, including all hard potentials with cutoff (that is when $s \geq 5$ ) and also soft potentials up to $s>13 / 5$.

One of the drawbacks of our proof is that instead of having a $L^{1}$ norm times a $L^{2}$ norm in the right-hand side of (1.5), we only get a $L^{2}$ norm to the square.

In Section 2, we deal with the case when $B$ is a tensor product (that is of the form (1.3)). Then, we present in Section 3 the case of general dependence for $B$ with a reasonable smoothness assumption.

The following notations will be used throughout the paper. For any $p \geq 1, q \geq 0, L_{q}^{p}\left(\mathbb{R}^{N}\right)$ is the weighted space embedded with the norm

$$
\|f\|_{L_{q}^{p}\left(\mathbb{R}^{N}\right)}=\left(\int_{v \in \mathbb{R}^{N}}|f(v)|^{p}(1+|v|)^{p q} d v\right)^{1 / p},
$$

and if $0<s<N / 2, \dot{H}^{s}\left(\mathbb{R}^{N}\right)$ is the homogeneous Sobolev space of functions $f$ of $L^{2 N /(N-2 s)}\left(\mathbb{R}^{N}\right)$ such that

$$
\widehat{f} \in L_{\mathrm{loc}}^{1}\left(\mathbb{R}^{N}\right) \quad \text { and } \quad|\xi|^{s} \widehat{f}(\xi) \in L^{2}\left(\mathbb{R}_{\xi}^{N}\right) .
$$

Its norm is given by

$$
\|f\|_{\dot{H}^{s}\left(\mathbb{R}^{N}\right)}=\left(\int_{\xi \in \mathbb{R}^{N}}|\widehat{f}(\xi)|^{2}|\xi|^{2 s} d \xi\right)^{1 / 2} .
$$

We shall use the two following formulas to compute some integrals on the sphere $S^{N-1}(N \geq 2)$. The first deals with functions which only depend on one component: for any function $\beta$ defined on ] $-1,1$,

$$
\int_{S^{N-1}} \beta\left(\omega_{N}\right) d \omega=\frac{2 \pi^{(N-1) / 2}}{\Gamma\left(\frac{N-1}{2}\right)} \int_{-1}^{1} \beta(u)\left(1-u^{2}\right)^{(N-3) / 2} d u .
$$


The second is concerned with the change of variables $\sigma=2(\xi \cdot \omega) \omega-\xi$, for a fixed $\xi \in S^{N-1}$. We have for any function $\varphi$ defined on $S^{N-1}$

$$
\int_{S^{N-1}} \varphi(\sigma) d \sigma=\int_{S^{N-1}} \varphi(2(\xi \cdot \omega) \omega-\xi)|2 \xi \cdot \omega|^{N-2} d \omega .
$$

Finally, constants will be denoted by $C$, or $C_{N}$ when they depend on the dimension $N$.

\section{The case of separated variables.}

We investigate here the properties of $Q^{+}$when

$$
B\left(\left|v-v_{*}\right|, \frac{v-v_{*}}{\left|v-v_{*}\right|} \cdot \sigma\right)=b\left(\left|v-v_{*}\right|\right) \beta\left(\frac{v-v_{*}}{\left|v-v_{*}\right|} \cdot \sigma\right),
$$

where $b$ and $\beta$ are Borel functions defined on $] 0, \infty[$ and $]-1,1[$ respectively. We consider the multidimensional case $N \geq 2$. Let us state the main result of this section.

Theorem 2.1. Assume that there exists $K \geq 0, \alpha \geq 0$ such that

$$
|b(x)| \leq K(1+x)^{\alpha}, \quad \text { for all } x>0,
$$

and that

$$
\beta \in L^{2}(]-1,1\left[,\left(1-u^{2}\right)^{(N-3) / 2} d u\right) .
$$

Then for any $f \in L_{1+\alpha}^{2}\left(\mathbb{R}^{N}\right), Q^{+}(f) \in \dot{H}^{(N-1) / 2}\left(\mathbb{R}^{N}\right)$ and

$$
\begin{aligned}
& \left\|Q^{+}(f)\right\|_{\dot{H}^{(N-1) / 2}\left(\mathbb{R}^{N}\right)} \\
& \quad \leq C_{N} K\|\beta\|_{L^{2}(]-1,1\left[,\left(1-u^{2}\right)^{(N-3) / 2} d u\right)}\|f\|_{L_{1+\alpha}^{2}\left(\mathbb{R}^{N}\right)}^{2} .
\end{aligned}
$$

In order to prove Theorem 2.1, let us define the operator $\widetilde{Q}^{+}$for functions of two variables $F\left(v_{1}, v_{2}\right), v_{1}, v_{2} \in \mathbb{R}^{N}$ by

$$
\begin{gathered}
\widetilde{Q}^{+}(F)(v)=\iint_{\substack{v_{*} \in \mathbb{R}^{N} \\
\sigma \in S^{N-1}}} F\left(\frac{v+v_{*}}{2}-\frac{\left|v-v_{*}\right|}{2} \sigma, \frac{v+v_{*}}{2}+\frac{\left|v-v_{*}\right|}{2} \sigma\right) \\
\qquad \cdot \beta\left(\frac{v-v_{*}}{\left|v-v_{*}\right|} \cdot \sigma\right) d \sigma d v_{*} .
\end{gathered}
$$


Proposition 2.2. For the linear operator (2.5), we have

i) If $\beta \in L^{1}(]-1,1\left[,\left(1-u^{2}\right)^{(N-3) / 2} d u\right)$, then for any $F \in L^{1}\left(\mathbb{R}^{N} \times\right.$ $\left.\mathbb{R}^{N}\right), \widetilde{Q}^{+}(F) \in L^{1}\left(\mathbb{R}^{N}\right)$ and

$$
\left\|\widetilde{Q}^{+}(F)\right\|_{L^{1}\left(\mathbb{R}^{N}\right)}
$$

$$
\leq \frac{2 \pi^{(N-1) / 2}}{\Gamma\left(\frac{N-1}{2}\right)}\|\beta\|_{L^{1}(]-1,1\left[,\left(1-u^{2}\right)^{(N-3) / 2} d u\right)}\|F\|_{L^{1}\left(\mathbb{R}^{N} \times \mathbb{R}^{N}\right)} .
$$

Moreover, (2.6) is an equality if $\beta$ and $F$ are nonnegative.

ii) If $\beta \in L^{2}(]-1,1\left[,\left(1-u^{2}\right)^{(N-3) / 2} d u\right)$, then for any $F \in L^{2}\left(\mathbb{R}^{N} \times\right.$ $\left.\mathbb{R}^{N}\right)$ such that $\left(v_{2}-v_{1}\right) F \in L^{2}\left(\mathbb{R}^{N} \times \mathbb{R}^{N}\right)$, the integral $(2.5)$ is absolutely convergent for almost every $v, \widetilde{Q}^{+}(F) \in \dot{H}^{(N-1) / 2}\left(\mathbb{R}^{N}\right)$ and

$$
\begin{aligned}
& \left\|\widetilde{Q}^{+}(F)\right\|_{\dot{H}^{(N-1) / 2}\left(\mathbb{R}^{N}\right)} \\
& \quad \leq C_{N}\|\beta\|_{L^{2}(]-1,1\left[,\left(1-u^{2}\right)^{(N-3) / 2} d u\right)}\|F\|_{L^{2}}^{1 / 2}\left\|\left(v_{2}-v_{1}\right) F\right\|_{L^{2}}^{1 / 2} .
\end{aligned}
$$

Let us postpone the proof of Proposition 2.2 and deduce Theorem 2.1 .

Proof of Theorem 2.1. Let us define

$$
F\left(v_{1}, v_{2}\right)=f\left(v_{1}\right) f\left(v_{2}\right) b\left(\left|v_{2}-v_{1}\right|\right) .
$$

Then, definitions $(1.2),(1.3)$ and $(2.5)$ yield $Q^{+}(f)=\widetilde{Q}^{+}(F)$. Now, by (2.2) we have

$$
\begin{aligned}
\left|F\left(v_{1}, v_{2}\right)\right| & \leq\left|f\left(v_{1}\right)\right|\left|f\left(v_{2}\right)\right| K\left(1+\left|v_{2}-v_{1}\right|\right)^{\alpha} \\
& \leq K\left|f\left(v_{1}\right)\right|\left|f\left(v_{2}\right)\right|\left(1+\left|v_{1}\right|+\left|v_{2}\right|\right)^{\alpha} \\
& \leq K\left|\left(1+\left|v_{1}\right|\right)^{\alpha} f\left(v_{1}\right)\right|\left|\left(1+\left|v_{2}\right|\right)^{\alpha} f\left(v_{2}\right)\right| .
\end{aligned}
$$

Therefore,

$$
\|F\|_{L^{1}} \leq K\|f\|_{L_{\alpha}^{1}}^{2}, \quad\|F\|_{L^{2}} \leq K\|f\|_{L_{\alpha}^{2}}^{2},
$$


and since

$$
\begin{aligned}
\left|\left(v_{2}-v_{1}\right) F\left(v_{1}, v_{2}\right)\right| \leq & \left|v_{1}\right|\left|F\left(v_{1}, v_{2}\right)\right|+\left|v_{2}\right|\left|F\left(v_{1}, v_{2}\right)\right| \\
\leq & K\left|\left(1+\left|v_{1}\right|\right)^{1+\alpha} f\left(v_{1}\right)\right|\left|\left(1+\left|v_{2}\right|\right)^{\alpha} f\left(v_{2}\right)\right| \\
& +K\left|\left(1+\left|v_{1}\right|\right)^{\alpha} f\left(v_{1}\right)\right|\left|\left(1+\left|v_{2}\right|\right)^{1+\alpha} f\left(v_{2}\right)\right|,
\end{aligned}
$$

we have also

$$
\left\|\left(v_{2}-v_{1}\right) F\right\|_{L^{2}} \leq 2 K\|f\|_{L_{\alpha}^{2}}\|f\|_{L_{1+\alpha}^{2}} .
$$

Therefore, we can apply Proposition 2.2.ii), and we get $Q^{+}(f)=\widetilde{Q}^{+}(F)$ $\in \dot{H}^{(N-1) / 2}$,

$$
\left\|Q^{+}(f)\right\|_{\dot{H}^{(N-1) / 2}} \leq C_{N}\|\beta\|_{L^{2}} K\|f\|_{L_{\alpha}^{2}}^{3 / 2}\|f\|_{L_{1+\alpha}^{2}}^{1 / 2}
$$

Finally, (2.4) follows since

$$
\|f\|_{L_{\alpha}^{2}} \leq\|f\|_{L_{1+\alpha}^{2}}
$$

Proof of Proposition 2.2. Estimate i) is easy with (1.8), and we only prove ii). Let us first assume that $F \in L^{1}\left(\mathbb{R}^{N} \times \mathbb{R}^{N}\right)$. We perform the change of variables

$$
\sigma=2\left(\frac{v-v_{*}}{\left|v-v_{*}\right|} \cdot \omega\right) \omega-\frac{v-v_{*}}{\left|v-v_{*}\right|}
$$

According to (1.9),

$$
\begin{aligned}
& \widetilde{Q}^{+}(F)(v)=\iint_{\substack{v_{*} \in \mathbb{R}^{N} \\
\omega \in S^{N-1}}} F\left(v-\left(v-v_{*}\right) \cdot \omega \omega, v_{*}+\left(v-v_{*}\right) \cdot \omega \omega\right) \\
& (2.14) \quad \cdot \beta\left(2\left(\frac{v-v_{*}}{\left|v-v_{*}\right|} \cdot \omega\right)^{2}-1\right)\left|2 \frac{v-v_{*}}{\left|v-v_{*}\right|} \cdot \omega\right|^{N-2} d v_{*} d \omega .
\end{aligned}
$$

Since by i) $\widetilde{Q}^{+}(F) \in L^{1}$, we can compute its Fourier transform, which 
is given by

$$
\begin{aligned}
& \widehat{\widetilde{Q}^{+}(F)}(\xi) \\
& =\iiint e^{-i \xi \cdot v} F\left(v-\left(v-v_{*}\right) \cdot \omega \omega, v_{*}+\left(v-v_{*}\right) \cdot \omega \omega\right) \\
& v, v_{*} \in \mathbb{R}^{N} \\
& \omega \in S^{N-1} \\
& \cdot \beta\left(2\left(\frac{v-v_{*}}{\left|v-v_{*}\right|} \cdot \omega\right)^{2}-1\right)\left|2 \frac{v-v_{*}}{\left|v-v_{*}\right|} \cdot \omega\right|^{N-2} d v d v_{*} d \omega \\
& =\iiint_{\substack{v_{1}, v_{2} \in \mathbb{R}^{N} \\
\omega \in S^{N-1}}} e^{-i \xi \cdot\left(v_{1}-\left(v_{1}-v_{2}\right) \cdot \omega \omega\right)} F\left(v_{1}, v_{2}\right) \\
& \cdot \beta\left(2\left(\frac{v_{1}-v_{2}}{\left|v_{1}-v_{2}\right|} \cdot \omega\right)^{2}-1\right)\left|2 \frac{v_{1}-v_{2}}{\left|v_{1}-v_{2}\right|} \cdot \omega\right|^{N-2} d v_{1} d v_{2} d \omega,
\end{aligned}
$$

by the usual pre-post collisional change of variables. Next we perform a change of variables in $\omega$, given by an orthogonal hyperplane symmetry which exchanges the unitary vectors

$$
\frac{v_{1}-v_{2}}{\left|v_{1}-v_{2}\right|} \quad \text { and } \quad \frac{\xi}{|\xi|} \text {. }
$$

We obtain

$$
\begin{aligned}
\widehat{\widetilde{Q}^{+}(F)}(\xi)= & \iiint_{\substack{v_{1}, v_{2} \in \mathbb{R}^{N} \\
\omega \in S^{N-1}}} e^{-i\left(\xi \cdot v_{1}-\left(v_{1}-v_{2}\right) \cdot \omega \xi \cdot \omega\right)} F\left(v_{1}, v_{2}\right) \\
& \cdot \beta\left(2\left(\frac{\xi}{|\xi|} \cdot \omega\right)^{2}-1\right)\left|2 \frac{\xi}{|\xi|} \cdot \omega\right|^{N-2} d v_{1} d v_{2} d \omega \\
= & \int_{\omega \in S^{N-1}} \widehat{F}(\xi-\xi \cdot \omega \omega, \xi \cdot \omega \omega) \\
& \cdot \beta\left(2\left(\frac{\xi}{|\xi|} \cdot \omega\right)^{2}-1\right)\left|2 \frac{\xi}{|\xi|} \cdot \omega\right|^{N-2} d \omega,
\end{aligned}
$$

with $\widehat{F}$ the Fourier transform of $F$ in both variables. Finally, we make the change of variables

$$
\sigma=2\left(\frac{\xi}{|\xi|} \cdot \omega\right) \omega-\frac{\xi}{|\xi|}
$$


and get according to (1.9)

$$
\widehat{\widetilde{Q}+(F)}(\xi)=\int_{\sigma \in S^{N-1}} \widehat{F}\left(\frac{\xi-|\xi| \sigma}{2}, \frac{\xi+|\xi| \sigma}{2}\right) \beta\left(\frac{\xi}{|\xi|} \cdot \sigma\right) d \sigma .
$$

Now, in order to estimate (2.15) we assume that $F \in C_{c}^{\infty}\left(\mathbb{R}^{N} \times \mathbb{R}^{N}\right)$, so that $\widehat{F}$ is smooth. This assumption can easily be relaxed by cutoff and convolution of $F$ to get (2.7) in the general case.

We have by Cauchy-Schwarz's inequality

$$
\begin{aligned}
\left|\widehat{\widetilde{Q}^{+}(F)}(\xi)\right|^{2} \leq & \int_{\sigma \in S^{N-1}}\left|\widehat{F}\left(\frac{\xi-|\xi| \sigma}{2}, \frac{\xi+|\xi| \sigma}{2}\right)\right|^{2} d \sigma \\
& \cdot \int_{\sigma \in S^{N-1}}\left|\beta\left(\frac{\xi}{|\xi|} \cdot \sigma\right)\right|^{2} d \sigma,
\end{aligned}
$$

and the last integral can be computed using (1.8),

$$
\begin{aligned}
\int_{\sigma \in S^{N-1}} \mid \beta\left(\frac{\xi}{|\xi|} \cdot \sigma\right) & \left.\right|^{2} d \sigma \\
& =\frac{2 \pi^{(N-1) / 2}}{\Gamma\left(\frac{N-1}{2}\right)} \int_{-1}^{1}|\beta(u)|^{2}\left(1-u^{2}\right)^{(N-3) / 2} d u .
\end{aligned}
$$

Then,

$$
\begin{aligned}
\int_{\sigma \in S^{N-1}}\left|\widehat{F}\left(\frac{\xi-|\xi| \sigma}{2}, \frac{\xi+|\xi| \sigma}{2}\right)\right|^{2} d \sigma \\
=\int_{\sigma \in S^{N-1}} \int_{r=|\xi|}^{\infty}-\frac{\partial}{\partial r}\left|\widehat{F}\left(\frac{\xi-r \sigma}{2}, \frac{\xi+r \sigma}{2}\right)\right|^{2} d \sigma d r \\
\leq \int_{\sigma \in S^{N-1}} \int_{r=|\xi|}^{\infty}\left|\widehat{F}\left(\frac{\xi-r \sigma}{2}, \frac{\xi+r \sigma}{2}\right)\right| \\
=\int_{|\eta|>|\xi|}\left|\widehat{F}\left(\frac{\xi-\eta}{2}, \frac{\xi+\eta}{2}\right)\right| \\
\cdot\left|\left(\nabla_{2} \widehat{F}-\nabla_{1} \widehat{F}\right)\left(\frac{\xi-\eta}{2}, \frac{\xi+\eta}{2}\right)\right| \frac{d \eta}{|\eta|^{N-1}},
\end{aligned}
$$


where $\nabla_{1} \widehat{F}$ and $\nabla_{2} \widehat{F}$ denote the gradients of $\widehat{F}$ with respect to the first and second variables. Therefore,

$$
\begin{aligned}
& \int_{\xi \in \mathbb{R}^{N}}|\xi|^{N-1} d \xi \int_{\sigma \in S^{N-1}}\left|\widehat{F}\left(\frac{\xi-|\xi| \sigma}{2}, \frac{\xi+|\xi| \sigma}{2}\right)\right|^{2} d \sigma \\
& \quad \leq \iint_{\xi, \eta \in \mathbb{R}^{N}}\left|\widehat{F}\left(\frac{\xi-\eta}{2}, \frac{\xi+\eta}{2}\right)\right|\left|\left(\nabla_{2} \widehat{F}-\nabla_{1} \widehat{F}\right)\left(\frac{\xi-\eta}{2}, \frac{\xi+\eta}{2}\right)\right| d \xi d \eta \\
& \quad=2^{N}\left\||\widehat{F}|\left|\nabla_{2} \widehat{F}-\nabla_{1} \widehat{F}\right|\right\|_{L^{1}\left(\mathbb{R}^{N} \times \mathbb{R}^{N}\right)} \\
& \leq 2^{N}\|\widehat{F}\|_{L^{2}\left(\mathbb{R}^{N} \times \mathbb{R}^{N}\right)}\left\|\nabla_{2} \widehat{F}-\nabla_{1} \widehat{F}\right\|_{L^{2}\left(\mathbb{R}^{N} \times \mathbb{R}^{N}\right)} \\
& =2^{N}(2 \pi)^{2 N}\|F\|_{L^{2}\left(\mathbb{R}^{N} \times \mathbb{R}^{N}\right)}\left\|\left(v_{2}-v_{1}\right) F\right\|_{L^{2}\left(\mathbb{R}^{N} \times \mathbb{R}^{N}\right)},
\end{aligned}
$$

and together with (2.16)-(2.17), we obtain (2.7).

REMARK 2.1. A slightly weaker version of Theorem 2.1 is still true when one deals with (not too) soft potentials (with the angular cutoff of Grad).

Namely, for a cross section satisfying assumption (2.1) with

$$
\beta \in L^{2}(]-1,1\left[,\left(1-u^{2}\right)^{(N-3) / 2} d u\right)
$$

and

$$
b(x)=x^{-\alpha}, \quad 0<\alpha<\frac{N}{2},
$$

for any $f \in L_{1}^{2 N /(N-\alpha)}\left(\mathbb{R}^{N}\right)$, we have that

$$
Q^{+}(f) \in \dot{H}^{(N-1) / 2}\left(\mathbb{R}^{N}\right)
$$

with

$$
\begin{aligned}
& \left\|Q^{+}(f)\right\|_{\dot{H}^{(N-1) / 2}\left(\mathbb{R}^{N}\right)} \\
& \quad \leq C_{N, \alpha}\|\beta\|_{L^{2}(]-1,1\left[,\left(1-u^{2}\right)^{(N-3) / 2} d u\right)}\|f\|_{L_{1}^{2 N /(N-\alpha)}}^{2} .
\end{aligned}
$$

Actually, defining

$$
F\left(v_{1}, v_{2}\right)=f\left(v_{1}\right) f\left(v_{2}\right)\left|v_{2}-v_{1}\right|^{-\alpha},
$$


we have

$$
\begin{aligned}
\|F\|_{L^{2}\left(\mathbb{R}^{N} \times \mathbb{R}^{N}\right)}^{2} & =\iint\left|f\left(v_{1}\right)\right|^{2}\left|f\left(v_{2}\right)\right|^{2}\left|v_{2}-v_{1}\right|^{-2 \alpha} d v_{1} d v_{2} \\
& \leq\left\||f|^{2}\right\|_{L^{r^{\prime}}}\left\||f|^{2} *|v|^{-2 \alpha}\right\|_{L^{r}} .
\end{aligned}
$$

We choose $r=N / \alpha$, so that

$$
\left\||f|^{2} *|v|^{-2 \alpha}\right\|_{L^{r}} \leq C_{N, \alpha}\left\||f|^{2}\right\|_{L^{N /(N-\alpha)}}
$$

and we obtain

$$
\|F\|_{L^{2}}^{2} \leq C_{N, \alpha}\left\||f|^{2}\right\|_{L^{N /(N-\alpha)}}^{2} .
$$

Therefore,

$$
\|F\|_{L^{2}\left(\mathbb{R}^{N} \times \mathbb{R}^{N}\right)} \leq C_{N, \alpha}\|f\|_{L^{2 N /(N-\alpha)}}^{2}
$$

and similarly

$$
\left\|\left(v_{2}-v_{1}\right) F\right\|_{L^{2}\left(\mathbb{R}^{N} \times \mathbb{R}^{N}\right)} \leq C_{N, \alpha}\|v f\|_{L^{2 N /(N-\alpha)}}\|f\|_{L^{2 N /(N-\alpha)}} .
$$

We conclude by applying Proposition 2.2.ii).

\section{The general case.}

We now concentrate on the case when $B$ is not a tensor product. The estimate is not as straightforward as in Section 2, and we have to make a regularity assumption on $B$. Moreover, we only treat here the three-dimensional case.

Theorem 3.1. Let $B$ be a continuous function from $] 0, \infty[\times[-1,1]$ to $\mathbb{R}$, admitting a continuous derivative in the second variable. We assume that

$$
|B(x, u)|+\left|\frac{\partial B}{\partial u}(x, u)\right| \leq K(1+x)
$$

for all $x>0$ and $u \in[-1,1]$. Then, for any $\varepsilon>0$, there exists $a$ constant $C_{\varepsilon}$ only depending on $\varepsilon$ such that for any

$$
f \in L_{1}^{1}\left(\mathbb{R}^{3}\right) \cap L_{(3+\varepsilon) / 2}^{2}\left(\mathbb{R}^{3}\right)
$$


$Q^{+}(f) \in \dot{H}^{1}\left(\mathbb{R}^{3}\right)$ with

$$
\left\|Q^{+}(f)\right\|_{\dot{H}^{1}\left(\mathbb{R}^{3}\right)} \leq C_{\varepsilon} K\|f\|_{L_{(3+\varepsilon) / 2}^{2}}^{2} .
$$

Proof. Since

$$
|B(x, u)| \leq K(1+x),
$$

the result of Proposition 2.2.i) ensures that the integral (1.2) defining $Q^{+}(f)$ is absolutely convergent for almost every $v$, and that $Q^{+}(f) \in$ $L^{1}\left(\mathbb{R}^{3}\right)$,

$$
\left\|Q^{+}(f)\right\|_{L^{1}} \leq 4 \pi K\|f\|_{L_{1}^{1}}^{2} .
$$

Therefore, we can compute the Fourier transform of $Q^{+}(f)$,

$$
\begin{gathered}
\widehat{Q^{+}(f)}(\xi)=\iiint_{\substack{v, v_{*} \in \mathbb{R}^{3} \\
\sigma \in S^{2}}} e^{-i v \cdot \xi} f\left(\frac{v+v_{*}}{2}-\frac{\left|v-v_{*}\right|}{2} \sigma\right) f\left(\frac{v+v_{*}}{2}+\frac{\left|v-v_{*}\right|}{2} \sigma\right) \\
\cdot B\left(\left|v-v_{*}\right|, \frac{v-v_{*}}{\left|v-v_{*}\right|} \cdot \sigma\right) d \sigma d v d v_{*} \\
(3.4) \quad=\iiint_{\substack{v, v_{*} \in \mathbb{R}^{3} \\
\sigma \in S^{2}}} e^{-i \xi \cdot\left(v+v_{*}-\left|v-v_{*}\right| \sigma\right) / 2} f(v) f\left(v_{*}\right) \\
\cdot B\left(\left|v-v_{*}\right|, \frac{v-v_{*}}{\left|v-v_{*}\right|} \cdot \sigma\right) d \sigma d v d v_{*},
\end{gathered}
$$

according to the pre-post collisional change of variables. Thus we obtain

$$
\widehat{Q^{+}(f)}(\xi)=\iint_{v, v_{*} \in \mathbb{R}^{3}} e^{-i \xi \cdot\left(v+v_{*}\right) / 2} f(v) f\left(v_{*}\right) D\left(v-v_{*}, \xi\right) d v d v_{*}
$$

where for any $w, \xi \in \mathbb{R}^{3} \backslash\{0\}$

$$
\begin{aligned}
& D(w, \xi) \\
& \quad=\int_{\sigma \in S^{2}} e^{i|w| \sigma \cdot \xi / 2} B\left(|w|, \frac{w}{|w|} \cdot \sigma\right) d \sigma \\
& \quad=\int_{s=-1}^{+1} e^{i|w||\xi| s / 2} \\
& \quad \cdot \int_{\varphi=0}^{2 \pi} B\left(|w|, s \frac{\xi}{|\xi|} \cdot \frac{w}{|w|}+\sqrt{1-s^{2}} \sqrt{1-\left(\frac{\xi}{|\xi|} \cdot \frac{w}{|w|}\right)^{2}} \cos \varphi\right) d \varphi d s,
\end{aligned}
$$


with spherical coordinates and

$$
s=\sigma \cdot \frac{\xi}{|\xi|} .
$$

Integrating by parts, we get

$$
\begin{aligned}
D(w, \xi)=- & \int_{s=-1}^{+1} \frac{2 e^{i|w||\xi| s / 2}}{i|w||\xi|} \\
& \cdot \int_{\varphi=0}^{2 \pi}\left(\frac{\xi}{|\xi|} \cdot \frac{w}{|w|}-\frac{s}{\sqrt{1-s^{2}}} \sqrt{1-\left(\frac{\xi}{|\xi|} \cdot \frac{w}{|w|}\right)^{2}} \cos \varphi\right) \\
& \cdot \frac{\partial B}{\partial u}\left(|w|, s \frac{\xi}{|\xi|} \cdot \frac{w}{|w|}\right. \\
(3.8) \quad+\sqrt{1-s^{2}} & \left.\sqrt{1-\left(\frac{\xi}{|\xi|} \cdot \frac{w}{|w|}\right)^{2}} \cos \varphi\right) d \varphi d s \\
+ & \frac{2 e^{i|w||\xi| / 2}}{i|w||\xi|} 2 \pi B\left(|w|, \frac{\xi}{|\xi|} \cdot \frac{w}{|w|}\right) \\
& -\frac{2 e^{-i|w||\xi| / 2}}{i|w||\xi|} 2 \pi B\left(|w|,-\frac{\xi}{|\xi|} \cdot \frac{w}{|w|}\right)
\end{aligned}
$$

and therefore

$$
\begin{aligned}
|D(w, \xi)| \leq & \frac{4 \pi}{|w||\xi|} K(1+|w|) \int_{-1}^{+1}\left(1+\frac{|s|}{\sqrt{1-s^{2}}}\right) d s \\
& +\frac{8 \pi}{|w||\xi|} K(1+|w|) \\
\leq & \frac{24 \pi}{|\xi|} K\left(1+\frac{1}{|w|}\right) .
\end{aligned}
$$

Coming back to (3.5) and using the variables

$$
z=\frac{v+v_{*}}{2}, \quad w=v-v_{*},
$$

we get

$$
\widehat{Q^{+}(f)}(\xi)=\int_{w \in \mathbb{R}^{3}} W(f)(w, \xi) D(w, \xi) d w
$$


where

$$
W(f)(w, \xi)=\int_{z \in \mathbb{R}^{3}} e^{-i z \cdot \xi} f\left(z+\frac{w}{2}\right) f\left(z-\frac{w}{2}\right) d z
$$

is a Wigner-type transform of $f$. Then, according to Cauchy-Schwarz's inequality, we get for any $\varepsilon>0$

$$
\begin{aligned}
&\left|\widehat{Q^{+}(f)}(\xi)\right|^{2} \leq \int_{w \in \mathbb{R}^{3}}|W(f)(w, \xi)|^{2}(1+|w|)^{3+\varepsilon} d w \\
& \cdot \int_{w \in \mathbb{R}^{3}}|D(w, \xi)|^{2} \frac{d w}{(1+|w|)^{3+\varepsilon}} \\
& \leq C_{\varepsilon} \frac{K^{2}}{|\xi|^{2}} \int_{w \in \mathbb{R}^{3}}|W(f)(w, \xi)|^{2}(1+|w|)^{3+\varepsilon} d w .
\end{aligned}
$$

Finally, using Plancherel's identity, we obtain

$$
\begin{aligned}
& \int_{\xi \in \mathbb{R}^{3}}|\xi|^{2}\left|\widehat{Q^{+}(f)}(\xi)\right|^{2} d \xi \\
& \leq C_{\varepsilon} K^{2} \int_{w \in \mathbb{R}^{3}}\left(\int_{\xi \in \mathbb{R}^{3}}|W(f)(w, \xi)|^{2} d \xi\right)(1+|w|)^{3+\varepsilon} d w \\
&= C_{\varepsilon} K^{2}(2 \pi)^{3} \\
& \cdot \int_{w \in \mathbb{R}^{3}}\left(\int_{z \in \mathbb{R}^{3}}\left|f\left(z+\frac{w}{2}\right) f\left(z-\frac{w}{2}\right)\right|^{2} d z\right)(1+|w|)^{3+\varepsilon} d w \\
&= C_{\varepsilon} K^{2}(2 \pi)^{3} \iint_{v, v_{*} \in \mathbb{R}^{3}}\left|f(v) f\left(v_{*}\right)\right|^{2}\left(1+\left|v-v_{*}\right|\right)^{3+\varepsilon} d v d v_{*} \\
& \leq C_{\varepsilon} K^{2}(2 \pi)^{3}\|f\|_{L_{(3+\varepsilon) / 2}^{2}}^{4},
\end{aligned}
$$

by the same estimate as in (2.9) and the proof is complete.

REMARK 3.1. As in Section 2, one could here also treat singular $B$ (in the first variable) if one allowed to replace the weighted $L^{2}$ norms of $f$ in (3.2) by suitable (weighted) $L^{p}$ norms, with $p>2$. 
REMARK 3.2. As in [10], one could deduce from Theorems 2.1 and 3.1 regularity properties for the homogeneous Boltzmann equation. Notice that such properties give also counterexamples. For example one can prove that if $f$ is the solution of the homogeneous Boltzmann equation and if $f(0)$ is not smooth (the exact smoothness considered here depends on the properties of $B$ ), then for any $t>0, f(t)$ will also not be smooth.

This behavior is completely opposite to that of the Boltzmann equation without angular cutoff ( $c f .[5],[8])$.

\section{References.}

[1] Andréasson, H., Regularity of the gain term and strong $L^{1}$ convergence to equilibrium for the relativistic Boltzmann equation. SIAM J. Math. Anal. 27 (1996), 1386-1405.

[2] Bobylev, A. V., Exact solutions of the nonlinear Boltzmann equation and the theory of relaxation of a Maxwellian gas. Teor. Math. Phys. 60 (1984), 280-310.

[3] Cercignani, C., The Boltzmann equation and its applications. Springer, 1988.

[4] Chapman, S., Cowling, T. G., The mathematical theory of non uniform gases. Cambridge Univ. Press, 1952.

[5] Desvillettes, L., About the regularity properties of the non cutoff Kac equation. Comm. Math. Phys. 168 (1995), 417-440.

[6] Grad, H., Principles of the kinetic theory of gases. Flügge's handbuch der Physik 12 (1958), 205-294.

[7] Lions, P.-L., Compactness in Boltzmann's equation via Fourier integral operators and applications, Parts I, II and III. J. Math. Kyoto Univ. 34 (1994), 391-461 and 539-584.

[8] Proutière, A., New results of regularization for weak solutions of Boltzmann equation. Preprint (1996).

[9] Truesdell, C., Muncaster, R., Fundamentals of Maxwell's kinetic theory of a simple monatomic gas. Academic Press, 1980.

[10] Wennberg, B., Regularity in the Boltzmann equation and the Radon transform. Comm. Partial Diff. Equations 19 (1994), 2057-2074. 
[11] Wennberg, B., The geometry of binary collisions and generalized Radon transforms. To appear in Arch. Rational Mech. Anal.

Recibido: 21 de septiembre de 1.996

Revisado: 21 de enero de 1.997

François Bouchut and Laurent Desvillettes Université d'Orléans et CNRS, UMR 6628

Département de Mathématiques

BP 6759

45067 Orléans cedex 2, FRANCE fbouchut@labomath.univ-orleans.fr desville@labomath.univ-orleans.fr 